\title{
31
}

\section{Production planning and control on the basis of control theory}

\author{
Hans-Peter Wiendahl and Jan-Wilhelm Breithaupt \\ Institute of Production Systems, University of Hanover \\ Callinstr. 36, 30167 Hannover, GERMANY, \\ Tel: +49-511-762-2440 Fax: +49-511-762-3814 \\ E-mail:wiendahl@ifa.uni-hannover.de
}

\begin{abstract}
Control theory provides excellent tools to analyse and control dynamic systems. Using the funnel model and the theory of the operating characteristic curve, a continuous model of a production system was developed. Based on this model two controllers were designed to control backlog and WIP. Simulation experiments have confirmed that this concept ensures the synchronisation of capacity and work. A suggestion on how to integrate the strategy into PPC on the planning level is discussed in the following. The objective of this approach is to develop the present open loop control realised in PPC into a closed loop control.
\end{abstract}

\section{Keywords}

Production model, Automatic production control, APC, PPC, Logistics, Continuous model

\section{INTRODUCTION}

The continuing structural changes in society and thereby, also in the production environment contain new challenges which companies have to face with innovative approaches in order to remain competitive in the future. These changes affect the categories: product, market, production location and process. A long term study published in 1993 by McKinsey illustrates that the international competition is based upon three essential pillars: price, quality and time (Rommel, 1993).

From the above, the factor, time, experiences growing importance. But also the logistical quality features, delivery time and delivery reliability, appear increasingly 
as differentiating criteria in the market. A further decisive point is the increasing demand for the flexibility with respect to the customer wishes.

Companies and research are therefore called upon to develop their own innovative ideas and to expand them to new tools and procedures. In the following a new perspective is pointed out under the catchword automatic production control (APC), which can be helpful in improving a companies competitiveness in the future. The aim of this approach is to design a self-controlling production model based on control theory.

A couple of models for production control have already been developed. They failed, due to a missing clear definition of the interdependencies between the reference and control variables (transmission functions). In this approach the transmission function of the system can be defined by using the logistic operating curve which has been developed at the Institute of Productions System at the University of Hanover.

\section{A CONTINUOUS MODEL OF A PRODUCTION SYSTEM}

\subsection{Logistic objectives}

The quality of the logistic performance is determined by lead time and schedule performance in the job shop as outlined in figure 1. On the other hand, loading of the production facilities and work-in-process (WIP) influence the profitability of the manufacturing process. From these partly conflicting requirements concerning the production planning and control (PPC) the four main objectives of the production process an be derived. Short lead time and low schedule deviation

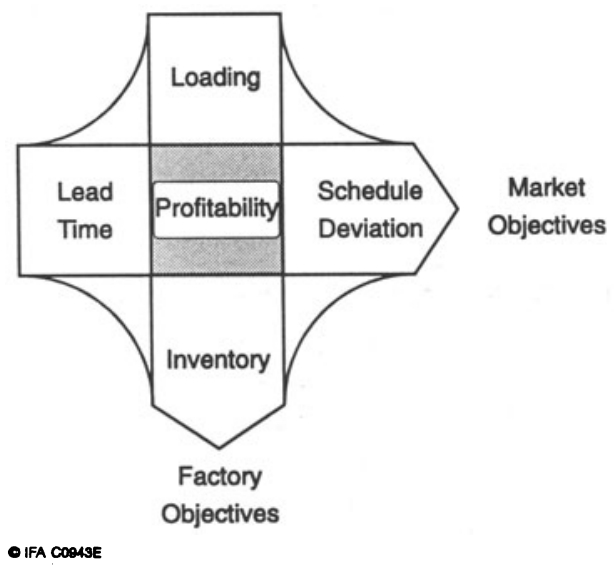

Figure 1 Logistic objectives in manufacturing 
represent the market-related objectives, whereas low inventory and high and steady loading of the work systems are the factory-related objectives.

In order to adjust the logistical objectives under the consideration of their mutual dependencies and the aspect of competitive production, there exists the demand to design a controllable production and order processing. While conventional PPC systems predominantly control (open-loop) and therefore a feedback is missing, a self-controlling process can be achieved by a closed-loop control by defining appropriate reference and correcting variables.

\subsection{The concept of a continuous production model}

Present day PPC systems differentiate between the planning and the implementation level. These views can also be applied to the production control. Due to the different characters of the levels, different models are needed. Single events are of interest on the implementation level. A more global view on the planning level makes the utilisation of a continuous model possible. This is desirable because the theory of control has a larger number of methods for continuous processes available. In addition, the planning level is responsible for the definition of the side constraints for attaining the goals on the operational level. The planning level of the production control is therefore the essential object to be viewed in the following (Petermann, 1995).

Various models for production control have already been developed. Most of them are based on a simple control loop. The feedback in today's PPC systems can be mentioned here as an example. The feedback is restricted to the closing of information circuits. The actual reaction remains with the system user. Such concepts are missing a clear definition of the control variables as well as a description of the relationship between effects. Thus the correcting variables can not be derived effectively. The deterministic models underlying this system merely describe reality statically and are not appropriate for depicting the dynamics of reality. Most of the systems are laid out to enforce the plans generated and show a strong static character because of this.

It is therefore necessary to develop a continuous production model that bases on closed-loop control technology. A continuous model of a production system can be developed with the aid of the funnel model and the logistic operating curve (Petermann, 1995). The underlying funnel model, suitable on the implementation level, depicts the individual events at a work system and represents a discrete model. The substitution from discrete to continuous models is successful, when the microscopical behaviour of the discrete individual process disappears behind the macroscopical. This is to be assumed for controlling on the planning level, because here the individual event is not of interest, but rather mean variables, like loading, WIP or lead time observed over a period of time. 


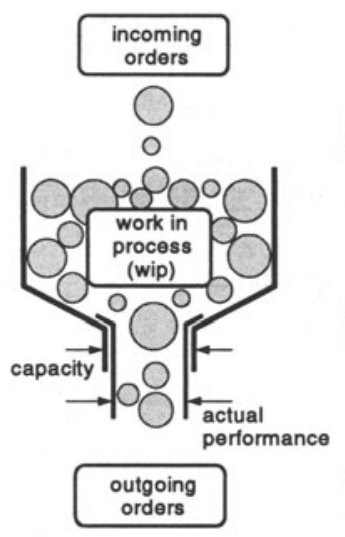

discrete funnel model of a work system

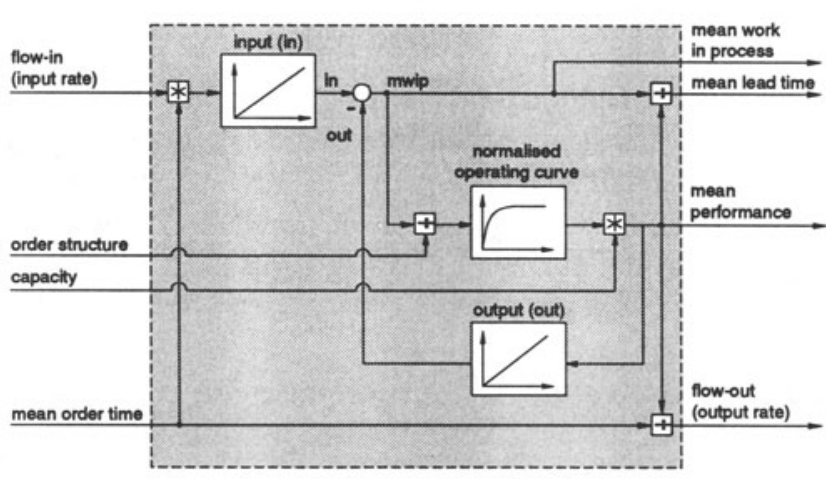

continuous model of a work system

- IFA D16540

Figure 2 Comparison of the input and output variables of discrete and continuous models

The input and output variables as well as a simple control loop of the continuous model are pictured in figure 2 on the right side. The incoming orders, measured in hours of work content in the funnel model, are converted to the input rate (dimension: orders per unit of time). Analogue to this, the same procedure is followed on the output side for the outgoing orders, which are then represented by the output rate. The various sizes of the orders, demonstrated as different sized balls in the funnel on the left side, are considered in the continuous model through the mean weighted operation time as a parameter of the order structure.

The other variables are comparable with the discrete model but are calculated as mean values. Inside the continuous model a simple control loop is depicted. With the aid of a time integral the input rate of the system is transformed into the input (IN). Analogue to this, the same procedure is followed for the output (OUT). The mean WIP (MWIP) is calculated as the difference between the input and output (summation point). The mean weighted lead time results from dividing the mean WIP by the output rate (funnel formula) (Wiendahl, 1995). The four variables mean performance, output rate, mean weighted lead time and mean WIP are the output variables of the system. The logistic operating curve forms the connection between the mean WIP, the mean weighted lead time and the mean performance with the aid of the capacity and the ideal minimal WIP (order structure) as input parameters (figure 3). Using the normalised version it describes the interdependence between utilisation (UT) and relative mean work in process (MWIP ${ }_{\text {rel }}$ ) which is the relation between mean WIP (MWIP) and minimum mean WIP (MWIP ${ }_{\text {min }}$ ) (Nyhuis, 1994). 


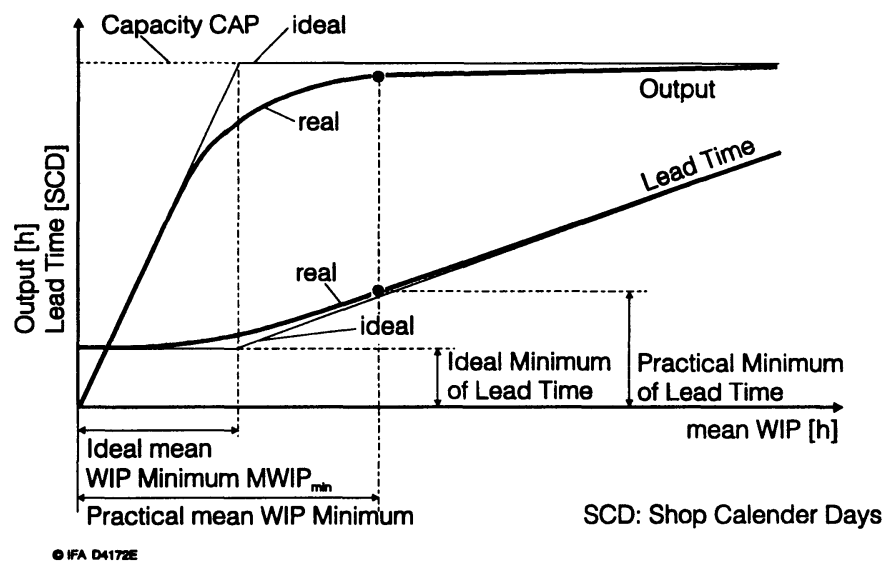

Figure 3 Interdependency between output, lead time and work-in-process (WIP) (Nyhuis, 1994)

The operating curve states that the production of a work system is independent of the WIP as long as every work system has a store of pending orders at all times. Then the output of the system is almost equal to its capacity. Only if the inventory is further reduced losses in production will occur due to interruptions in the material flow. On the other hand, the lead time decreases in proportion with the WIP until the bending point is reached. Beyond this point the lead time cannot be reduced further, because it is limited by the sum of the operation and minimum transportation time (idealised minimum of lead time). The idealised mean WIP minimum (MWIPmin) represents the WIP level that is necessary to run the system under idealised conditions, assuming that no arriving order has to wait. This cannot be found in practice, so the realistic curves differ from the idealised ones shown in figure 3 , even though it is possible to calculate these realistic curves for most of the job shop production (Nyhuis, 1994). Therefore the operating curve is an excellent solution to constitute the transmission function of the continuous model.

\subsection{Model evaluation}

The transition from a discrete process to a continuous process is difficult. Is this transition fundamentally allowed? When does the microscopic behaviour of the single event (here: the processing of a operation) stand behind the macroscopic behaviour of the system with reference to the viewed input and output variables? To be able to answer these questions, extensive model evaluations have been necessary. 
For the discrete simulations performed within the scope of the model evaluation, the event-controlled production simulator PROSIM II, which was developed at the Institute for Production Systems (IFA), was used. The comparison simulation for

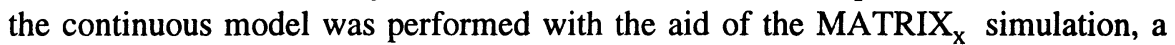
special simulation software for the support of cybernetic systems. The ability to transfer the discrete model to the continuous model was verified based on numerous test runs (Petermann, 1995).

\section{CONTROLLER DESIGN}

\subsection{Definition of reference and control variables}

The design of a controller concept has to be divided into two different steps. First of all, it is necessary to define the controllable output variables under consideration of the input variables. Afterwards, the linkage of the controller has to be designed. With the aid of the models presented two controllers for a work system were able to be modelled. The input and output variables of the continuous model have been discussed in section 2.2. The variables output performance, WIP and lead time, are linked through the funnel formula. Therefore only two of the variables are controllable at one time.

The essential task of a work system is to allocate the required performance to process the system load (production schedule). For this reason the output performance, respectively, the output rate attains importance. When viewing the system's output performance, the actual output rate is of less importance. The question of interest is whether the planned work is finished by a certain date. The difference between the planned sum and the actual output is defined as the backlog of the system. The backlog of the system thus becomes the most important control variable for monitoring the output performance of the production system. In order to utilise a clear definition, the newly developed controller is therefore named backlog controller instead of output controller. The capacity is used here as a correcting variable of the system. Fig. 4 shows the concept of a backlog controller.

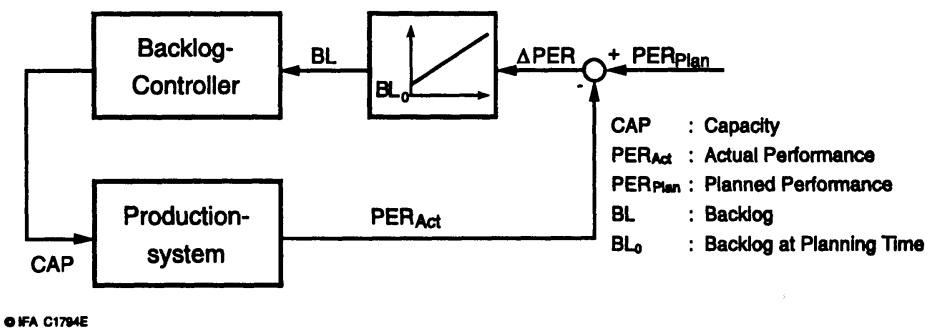

Figure 4 Concept of an automatic backlog controller 


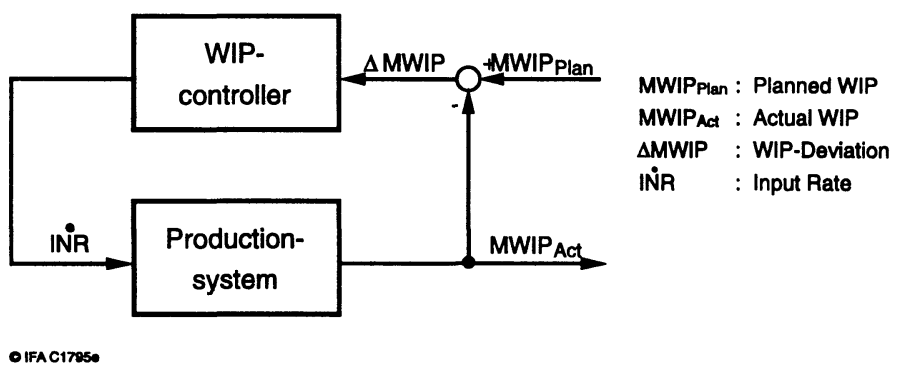

Figure 5 Concept of an automatic WIP controller

The planned performance is the reference variable. The difference between the actual and the planned performance is integrated. The result is the above mentioned backlog. Referring to the actual backlog the following backlog controller adjusts the required capacity of the work system. Because in reality it is impossible to adjust the capacity immediately, a dead time between the request for capacity and the following allocation was introduced (Petermann, 1995).

After defining the backlog as a control variable, a decision whether the lead time or the WIP shall be controlled is necessary. A controllable order processing based on production scheduling supports the implementation of the lead time as control variable. But two basic problems derive from an implementation of the lead time: firstly, the measurement of the variable is difficult; secondly, the value of the lead time is limited at the lower end. This is problematical if the reference value of the lead time falls short of this limit. The mean WIP as control variable is not limited. Moreover its measurement is easier and more precise. Therefore it is obvious to use WIP control.

The main task of the WIP controller (figure 5) is to set the system to an operating point on the logistic operating curve that was defined within the scope of the production planning. The planned WIP is the reference variable. Referring to the difference between planned and actual WIP the WIP controller adjusts the input rate of the production system (Petermann, 1995).

\subsection{Combination of the backlog and WIP controller}

The calculation of the required capacity for the next period depends on the operating point on the logistic operating curve of the work system. The developed concept for the backlog controller can only catch when the planned utilisation of the system is reached since otherwise backlog does not arise. The WIP controller is suitable for this task. The principle working method of both controllers can be compared with the conventional production control methods. In the case of increasing backlog in a production system, it is useful to increase the capacity. If 


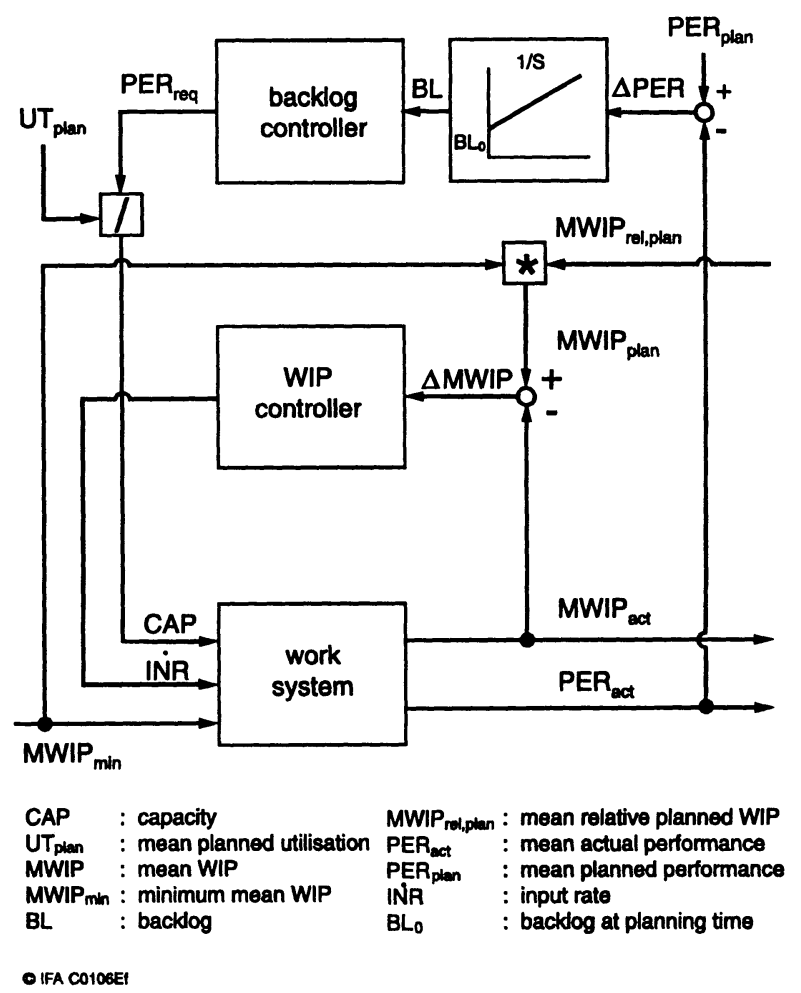

Figure 6 Concept of a combined WIP and backlog controller

the throughput time keeps growing, the line-up (queue) in front of the work system can be diminished through reducing the input rate of the system. The operating characteristic curve is a qualified tool for combining both concepts with each other. Simulation experiments have confirmed that this approach guarantees the synchronisation of capacity and work. Figure 6 shows the integration of both controllers in a controller concept.

The first step is to decide on which operating point on the characteristic curve the system should be driven. This can be done by deciding which utilisation the system ought to reach. For important or expensive systems this value must be higher than for the other systems. So the backlog control loop is enhanced by calculating the planned output first and then using the planned utilisation for determining the necessary capacity. The relative WIP is multiplied by the actual minimum mean WIP (MWIPmin). This results in the planned mean WIP.

Deviations between the planned and realised performance of the system are integrated over time and defined as the backlog of the system. The backlog controller calculates the planned performance for the next period which is divided by the planned utilisation. The result represents the corrected capacity of the 
system. The planned relative mean WIP is multiplied by the actual minimum mean WIP. This delivers the planned mean WIP as the reference value of the WIP controller for the next period. This value is compared with the actual mean WIP in the system and by deviations the WIP controller corrects the input rate.

The whole concept and the control design were created by using control theory methods and simulations. Furthermore simulations were used to evaluate the control design. Those simulation experiments testify that the described strategy fulfils the requirements that were postulated in the beginning with a high grade: Material (work) and capacity always come together at the same time.

The market often requires short term changes for orders to be carried out without being planned in the production program. This leads to more or less orders which must be executed unplanned but with high priority. In the upper part Figure 7 illustrates the effect of such an urgent order on a balanced system without control, in the lower part, with the described control system installed. The unplanned order with a work content of $10 \mathrm{~h}$ arrives on SCD 26. As it is called an urgent order it is processed immediately after its arrival. Due to this, WIP rises by $10 \mathrm{~h}$ to $17 \mathrm{~h}$ and backlog comes up because planned work cannot be carried out. Because of its low time constant the system is able to reduce both backlog and WIP to the initial level relatively fast (34 SCD).
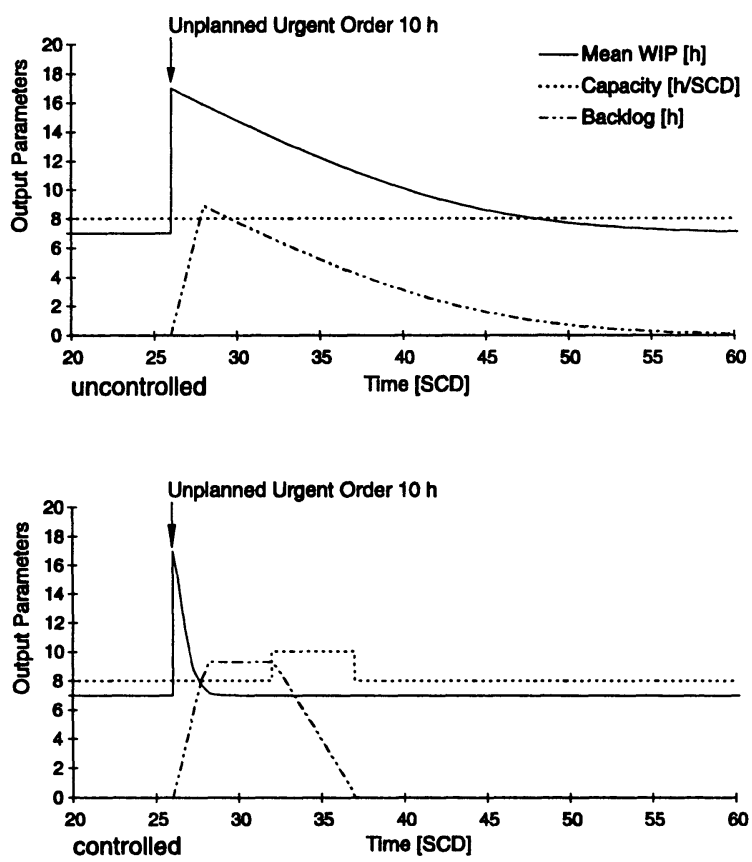

- ifa csosoe

Figure 7 Impact of an unplanned urgent order on the control parameters 
The controlled system reacts completely different. As the first measure the WIP controller reduces the input rate to decrease WIP to the planned level. The backlog controller works periodically every 5 days and corrects the capacity after 2 days dead time at SCD 32 exactly to that value that is necessary to decrease the backlog to zero during the following period. At the same time the WIP controller increases the input rate so there is enough work in the system.

This demonstrates that work that cannot be performed is not released until there is sufficient capacity available to carry it out. Capacity and work come together at the same time keeping throughput times at the planned level and compensating disturbances between load and capacity. The quality of this process in a system with this control strategy installed is independent from the initial operating state of the production system. The uncontrolled system behaviour though becomes worse with higher utilisation as previously mentioned.

\section{INTEGRATION OF THE DEVELOPED CONTROLLERS IN A PPC ENVIRONMENT}

PPC systems of tomorrow will certainly not be based on integrated circuits. From today's view, three areas can be identified in which changes in PPC could arise from the previously mentioned approach.

To begin with, it is to be expected that new elements enlarge the understanding of the process and its rules of behaviour, as was shown in the example above. Beyond this, it is quite imaginable that improved techniques and algorithms for the planning and enforcing of order processing can be developed on the basis of these models. It can be determined quite definitely, that a rethinking of the function architecture in PPC is necessary if a controlled process is to be attained. The following are new characteristics of a process governed by controlling: (1) short-term realisation of changing goals with respect to time and locality (guidance behaviour), (2) compensation of occurring disturbances (disturbance sensitivity) and (3) quickest possible attainment of a state of balance (stability).

A proposal for such a functional concept is shown in figure 8. The levels, planning and operation, are kept in the usual form. Main tasks are named within the levels with respect to production control. Three cross-section functions are mentioned alongside. The entire architecture must demonstrate a continual transparency between the control variables and the parameters used in the functions. This can be realised most consistently when all implemented procedures are based as much as possible on the same models.

The WIP controller affects the input side through the order release; the backlog controller has to guarantee the capacity. The higher the capacity flexibility of a production is, the more the output performance control stands out over the WIP control. Capacity flexibility can be described on the whole by the length of time, in which a change of capacity can be realised and by the limits within which this 


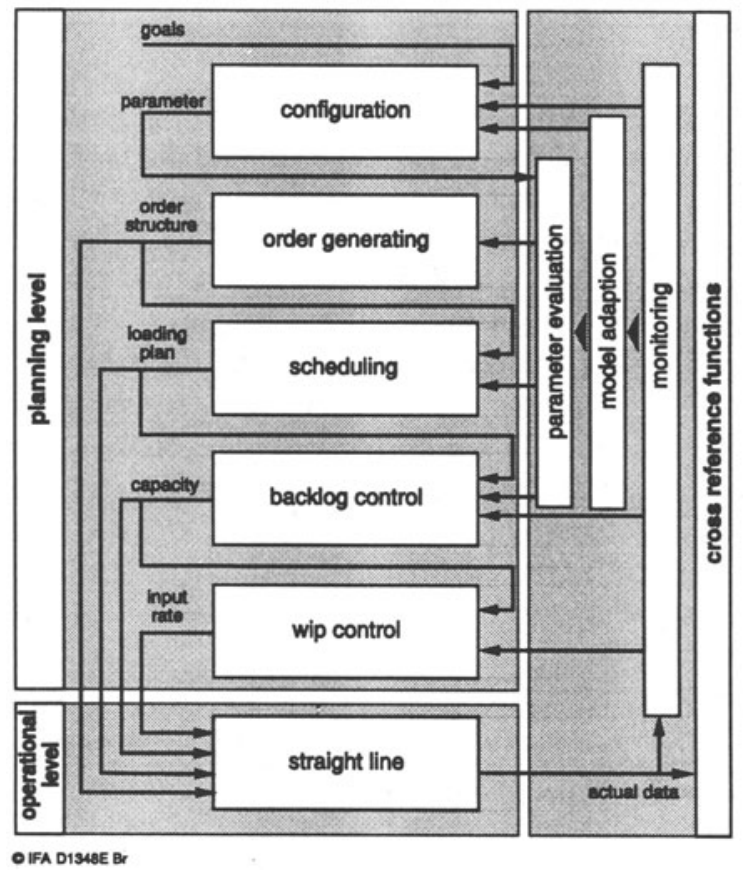

Figure 8 Functional architecture of an automatic production control system

change can range. The scheduling and generating of orders are to be understood as correcting variables. The order generation results in the corresponding order structure which most likely arises in the work system. The scheduling sets up a dynamic loading plan for the work system and determines beyond this, the planned start dates.

The configuration represents a function, which is largely unknown in the classical field of PPC. Its task is to secure the commitment of consistent and also reachable goals. On top of this, it represents the connecting link between goals and process parameters which brings about the transparency demanded above.

At the latest in the configuration the total system does not work automatically, but rather with a strong binding to the human being. Other important cross-section functions are the model adaptation and the parameter evaluation. The latter has the task of monitoring the parameter adjustments while in operation and if necessary, pointing out inconsistencies that arise. These inconsistencies can arise from disturbances or from the process itself. The model adaptation has to guaranty a continual and highest possible automated adaptation of the models used, because even controlling can not function when the underlying control path model does not comply with reality anymore. All three cross-section functions work closely together and can mutually support each other (Petermann, 1995). 


\section{SUMMARY}

A production process control approach contributes to the development of new algorithms and therefore, ultimately also to the development of new function architectures for PPC. With that, the possibility of a self-controlling, dynamic process, which can respond quickly to changing environmental conditions, is given. The validity of the continuous model as well as the controller concept was evaluated for a single work system (a single machine, a machine group, a cost centre or an entire factory). The model itself is already suitable for the connection of several work systems by the material flow. The extension of the controller concept to describe the interaction of different work systems is subject to further studies at the Institute for Production Systems.

\section{REFERENCES}

Nyhuis, P. (1994). 'Logistic operating curves - a comprehensive method of rating logistic potentials'. Paper at the conference "EURO XII /OR 36", University of Strathclyde, Glasgow, United Kingdom.

Rommel, G. 1993). 'Einfach überlegen'. Schäffer Poeschel Verlag Stuttgart.

Petermann, D. (1995). Modelbasierte Produktionsregelung, Fortschrittsberichte, Reihe 20, Nr. 193, VDI, Düsseldorf

Wiendahl, H.-P. (1991). Modellbasiertes Planen und Steuern reaktionsschneller Produktionssysteme, GfMT, München.

Wiendahl, H.-P. (1995). Load-orientated manufacturing control, Springer, Berlin.

\section{BIOGRAPHY}

Hans-Peter Wiendahl graduated from the University of Aachen as a DiplomEngineer. 1967-1970 he was research assistant at the Laboratory for Machine Tools and Workshop Management, before graduating as Dr.-Ing.. 1972-74 he was employed at a big industrial firm and stayed there as head of the technical department, Paper Machine Branch, until 1979 when he was appointed as full time professor and director of the Institute of Production Systems (IFA), University of Hanover. He is referee for various research associations and a full member of CIRP since 1989. In 1997 he became chairman of the German Society of Production engineering.

Jan-Wilhelm Breithaupt graduated 1994 from the University of Hanover. In 1995 he was Trainee at one of the leading industrial groups in Germany. Since 1996 he is research assistant at the Institute of Production Systems (IFA) at the University of Hanover. His field of research is production control. 